\title{
Relationships between neuronal cell adhesion molecule and LHRH neurons in the urodele brain: a developmental immunohistochemical study
}

\author{
S. Gianola, P. Clairambault ${ }^{1}$, and M.F. Franzoni
}

Dipartimento di Biologia Animale e dell'Uomo, Laboratorio di Anatomia Comparata, Università degli Studi di Torino, Via Accademia Albertina, 13. 10123 Torino (Italy); 'Laboratoire d'Anatomie Comparée, Université PARIS VII, Paris cedex 5, France

Key words: neural cell adhesion molecules, polysialic acid, LHRH-containing neurons, immunohistochemistry, development, cell migration, urodele amphibians

\section{SUMMARY}

Polysialic acid (PSA), a homopolymer attached to neural cell adhesion molecule (NCAM) is considered a major hallmark of vertebrate cell migration. We studied the distribution of PSA-NCAM by immunohistochemistry, during brain development, in two urodele amphibians, Pleurodeles waltl and the neotenic newt Ambystoma mexicanum. In both species a gradual increase of immunolabelling was observed throughout the brain from developmental stage 30 to stage 52. At the onset of metamorphosis, some differences became evident: in Pleurodeles immunostaining was gradually restricted to the olfactory system while in Ambystoma, PSA-NCAM maintained a more extended distribution (for example throughout the telencephalic walls) suggesting, for the brain of this latter species, a rather preserved neuronal plasticity. The aim of the present work was to correlate the above described PSA-NCAMimmunoreactivity (IR) with the distribution of luteinizing hormone-releasing hormone (LH-RH) containing neurons, which represent a well known example of neural elements migrating from the olfactory placode. LHRH-IR, undetectable till stage
30, was later found together with PSA-NCAM-IR in both the olfactory system and septo-hypothalamic areas. Such observations further support a role of PSA in providing a migration route toward the establishment of a part, at least, of the urodele LHRH system. The possible functional meaning of the LHRH-containing neurons localized between dorsal and ventral thalamus of Ambystoma, never reported before in this area, almost devoid of PSANCAM-IR, is discussed.

\section{INTRODUCTION}

The brain of Urodeles has often been considered as primitive when compared with that of Anurans, being characterized by a compact lamina of periventricular gray matter and few clusters of migrated cells or nuclei (Becker et al., 1993). Such primitivity was correlated with a reduced cellular migration during embryonic development, leading to a secondary simplification or retention of larval traits in the adult brain, also called paedomorphosis (Roth $e t$ al., 1993). Recently, emphasis has been placed on one type of embryonic neuronal molecule involved 
in the cellular migration process: the neural cell adhesion molecule or NCAM, which is a phylogenetically conserved membrane protein occurring in the nervous tissues of all groups of vertebrates (Hoffman et al., 1984; Becker et al., 1993). The polysialylated NCAM (PSA-NCAM) represents an important form of post-translational modification of NCAM, involving glycosylation, and characterized by a strong reduction in binding rates (Hoffman and Edelman, 1983). The higher percentage of this isoform during the early period of cell migration is consistent with a role in promoting plasticity. In spinal cord motor neurons, for example, the highest level of PSA-NCAM is detected at the time axons are sorting toward the muscles (Fields and Itoh, 1996) while a decrease in PSA correlates with areas of increased morphological stability (Sunshine et al., 1987, Fields and Itoh, 1996). Therefore, the length of the chain of sialic acid residues is developmentally regulated: during late embryonic life and early postnatal periods, PSA-NCAM is abundant throughout the nervous system and then rapidly decreases with the establishment of neuronal connections (Sunshine et al., 1987; Shults and Kimber, 1992). Nevertheless, PSA-NCAM was found to persist in some regions of the adult rodent brain characterized by neuronal plasticity, such as the hypothalamo-neurohypophysial system (Theodosis et al., 1991; Soares et al., 2000) and the subependymal layer (Bonfanti and Theodosis, 1994).

Data concerning spatial and temporal variations of CAMs (total CAM, NCAM-180 and PSA-NCAM) during ontogeny of the amphibian brain are scarce with the exception of Xenopus brain (Sunshine et al., 1987) and the optic tectum of both salamanders and frogs (Becker et al., 1993) where NCAM expression patterns do not substantially differ.

In the present paper, the distribution of PSANCAM-IR was studied during brain development in two salamanders, the Spanish newt Pleurodeles waltl and the axolotl Ambystoma mexicanum. This latter is an aquatic species characterized by reproduction during the larval stages (neoteny). Our analysis was performed from early developmental stages till metamorphosis as well as in some juvenile and adult specimens.

LHRH containing neurons are a well known example of migrating cells which have been exhaustively investigated in both larval and adult Anurans (see for example King et al., 1994; Northcutt and
Muske, 1994; Hayes et al., 1994; D'Aniello et al., 1995). In these amphibians, developmental studies (Muske, 1993) have shown that LHRH neurons have at least two origins: the olfactory placode, which gives rise to the terminal nerve-septo-preoptic LHRH system, and a nonplacodal precursor giving rise to a second LHRH neuron subset localized in the caudal diencephalon and midbrain. Moreover, two different molecular forms of LHRH (mammalian-like and chicken II-LHRHs) were, respectively, expressed by the preoptic area and the caudal diencephalon/mesencephalon of both urodeles (Muske et al., 1994) and anurans (D'Aniello et al., 1995; Collin et al., 1995).

By contrast, the only data concerning development of urodele LHRH neurons were reported by Murakami et al. (1992), in the red spotted newt Cynops pyrrhogaster) and by Northcutt and Muske (1994, in Ambystoma mexicanum).

Therefore the study of correlations between NCAM-PSA- and LHRH-IRs during development, in both the olfactory system and brain proper, might provide further contributions to better understanding the role of PSA-NCAM in promoting LHRH neuron settlement.

\section{MATERIALS AND METHODS}

\section{Animals}

The study protocol complied with Italian legislation on the care and use of animals in research.

Larvae, juvenile and adult specimens of Ambystoma mexicanum (adult $\mathrm{n}=1$ ) and Pleurodeles waltl (adult $\mathrm{n}=1$ ) were taken from breeding colonies reared in the laboratory. Developmental stages (from 22 through 52) were established according to Gallien and Durocher (1957), with some adaptations for Ambystoma. Juvenile and adult specimens were 9 months to 3 years old. All animals were anaesthetized with $1 \%$ methanesulfonate of tricaine in t.w. (MS222, Sandoz, Switzerland). Brains of the adult and juvenile specimens were removed and quickly fixed in Bouin's or Susa's solutions overnight at room temperature. The larvae $(n=40$ of Ambystoma, from stage 22 through stage 52 and $n=$ 45 of Pleurodeles, from stage 28 through stage 52), were immersed in the above fixatives ( 2 hoursovernight). Tissues were then washed, dehydrated through graded alcohols and n-butanol and embed- 
ded in paraffin. Two series of $12 \mu \mathrm{m}$ thick sections, following coronal and sagittal planes, were cut on a rotary microtome, mounted on glass slides and stored in boxes at room temperature.

\section{Immunohistochemistry}

Sections were deparaffinated, washed in phosphate buffered saline (PBS) and incubated in normal goat serum (1:50 in PBS) for 30 min to reduce non specific staining. After a rapid wash in PBS, sections were incubated, in a moist chamber in the dark at $4{ }^{\circ} \mathrm{C}$ for $18-24$ hours, in one of the following primary antibodies:

1. mouse IgM monoclonal anti PSA-NCAM, raised against the capsular polysaccharides of meningococcus group B that share $\alpha$-2,8-PSA residues with PSA-N-CAM (Rougon et al., 1986), 1:1000 dilution (courtesy of Dr. G. Rougon, Marseille, France);

2. rabbit polyclonal anti mammalian-LHRH (mLHRH), 1:5000 dilution (courtesy of Dr R. Benoit, Montreal, Canada);

3. rabbit polyclonal anti chicken II-LHRH (c IILHRH), 1:1000 dilution (courtesy of Dr F. Vandesande, Leuven, Belgium).

Immunoreaction was revealed with the avidinbiotin-horseradish peroxidase procedure (Vectastain ABC kit, Vector Labs, CA, USA) by using the diaminobenzidine (DAB, Sigma Chemical Co.) as the chromogen.

Specificity of the primary antibodies was tested in previous investigations (for the anti PSANCAM see Rougon et al., 1986; for the anti mLHRH see Schwanzel-Fukuda et al., 1992 and for the anti c II-LHRH see van Gils et al., 1993).

The reactions were considered to be specific when the omission of the primary antibodies totally abolished immunostaining.

\section{RESULTS}

In the urodele larvae, the closure of the neural tube occurred around stage 21 . In the same period the optic vesicles became enlarged and nasal pits, ear vesicles together with lateral line placodes began to be observable. By stage 23, the prosencephalic vesicle has been subdivided into telencephalon and diencephalon by a deep cephalic flessure. By stage 27, the principal encephalic compartments were well recognizable in the brain of the two species under study.

The following observations refer to both Ambystoma and Pleurodeles; nevertheless the discrepancies observed in the two species are properly reported.

From developmental stage 22 through stage 27, both LHRH- and PSA-NCAM-IRs were absent in the epithelium of the olfactory pits and any area of the brain in both species.

\section{PSA-NCAM-IR}

The first appearance of PSA-NCAM-IR could be coincident to stage 28 (a bit later in the Axolotl) when patches of very intense immunolabelling were seen within the prosencephalon (Fig. 1A, stage 30) and the hindbrain. Cells of the olfactory pit were also stained. From stages 31/32 PSANCAM-IR became very abundant throughout the brain. A bit later (stage 36) the PSA-NCAM-IR began to assume its peculiar staining pattern in outlining the plasma membranes of the olfactory epithelium and of some cell bodies in the adjacent olfactory bulb (Fig. 1B). By stage 38, a number of cells showing the characteristic PSA-NCAM staining was localized in some selected prosencephalic areas such as the dorsomedial pallium of the telencephalon, plus some centers mainly related to olfactory function, such as olfactory bulbs, olfactory tracts and nuclei, amygdala, habenulae and habenular commissure of the diencephalon (Fig. 1C,E). The same type of immunolabelling was shown by the gray matter of the optic tectum and tegmentum of the mesencephalon (Fig. 1D).

Up to developmental 50-52 stages, the intensity and extension of the PSA-NCAM-IR were gradually increasing throughout the brain of both species. In particular, a number of distinct fiber bundles became heavily immunostained, such as the stria medullaris (well appreciable in the neuropil of the habenular nuclei, see for example Fig. $1 \mathrm{E})$ and the nerve terminal roots which were intermingled with clusters of immunopositive cells in the septal area of the basal telencephalon (Fig. $1 \mathrm{~F})$. Immunopositive bundles were also seen in the preoptic area and the infundibular wall of the caudal hypothalamus (Fig. 3F).

At the onset of metamorphosis (stage 56), some differences became relevant between the two species: in Pleurodeles immunostaining was pro- 

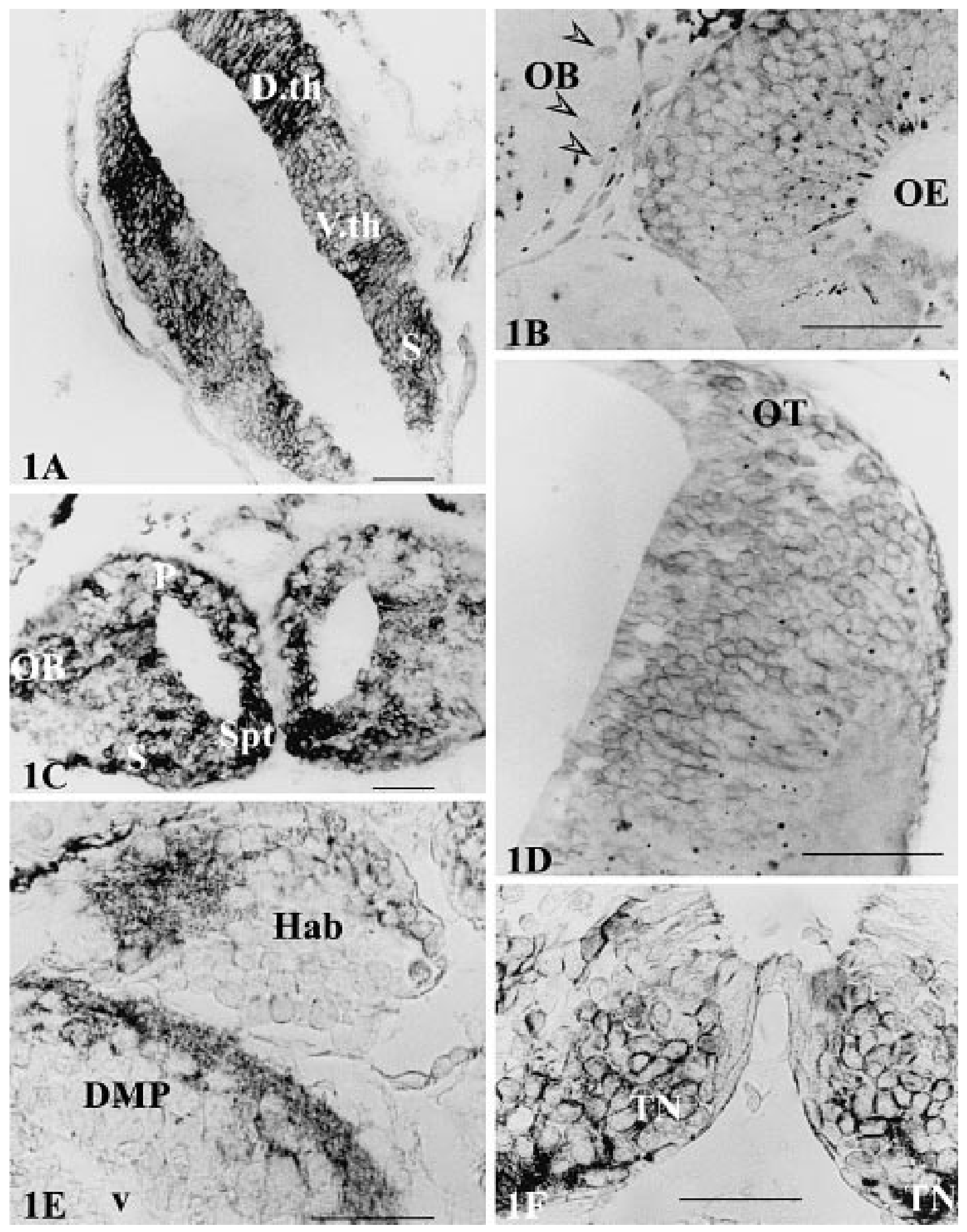
gressively restricted to the olfactory system. By contrast, in Ambystoma, PSA-NCAM-IR persisted in the fiber bundles forming the terminal nerve (Fig. 2A), in several cells in the dorsal pallium (Fig. 2B) and in the amygdala of the telencephalon, and in the periventricular gray of the preoptic recess. A number of stained cell bodies was also detected in the ependymal and subependymal layers (Fig. 2B,C) of the thalamus and suprachiasmatic hypothalamus. Interestingly, some immunopositive cells were found within the paraventricular organ (PVO, Fig. 2C) of the hypothalamus.

\section{LHRH-IR}

Since in the adult brain of the salamander Taricha granulosa were reported (Muske et al., 1994) two different forms of LHRH (mammalian-like and chicken II-LHRHs) expressed by different cell populations and axonal pathways, we have used two anti LHRH antibodies, one raised against the mammalian LHRH and the other one against the chicken II-LHRH, to evaluate possible differences in the expression of the two forms during development.

At stage 30, only m-LHRH-immunostaining was detected in the olfactory system (olfactory epithelium, fiber bundles in the olfactory nerve, cells and fibers in both the main and accessory olfactory bulbs) and hypothalamus. By stage 38 (after incubation with both anti m-LHRH and anti cII-LHRH antibodies) LHRH immunopositive neurons and fibers were observed in the posterior tubercle, mesencephalic tegmentum, periventricular gray of the optic tectum and pretectum (Fig. 3A), PVO and dorsal and ventral infundibular nuclei (NID and NIV). Only in Ambystoma, and for a limited period (stage 39), a bilateral small group of m-LHRH immunopositive tufted neurons sending their axons in the ventrolateral direction were found in the deepest layers of the periventricular gray, at the boundary between dorsal and ventral thalamus (Figs. 3B,C). Clusters of strongly labelled cells and terminals were observed in the dorsomedial (anterior olfactory nucleus) and ventromedial wall of the telencephalic hemispheres (Fig. 3D), septum and basal preoptic area. After immunostaining of serial sections, PSA-NCAM and m-LHRH-IRs were closely distributed in the ventral wall of the tuberal hypothalamus (Figs. 3E, 3F).

In juvenile and adult specimens, immunostaining was detected in numerous cells of the olfactory mucosa, in the glomerular layer and in a few cells of the adjacent olfactory bulb (Fig. 4A). Some positive cells were observed in the accessory bulb (Fig. 4B). An intense cII-LHRH-IR was shown in the basal telencephalon, preoptic area, tuberal hypothalamus, posterior tuberculum and ventral tegmentum of the mesencephalon (Fig. 4C).

\section{DISCUSSION}

In previous immunohistochemical studies, the description of PSA-NCAM-IR in developing amphibian brain was limited to the Xenopus brain (Sunshine et al., 1987) and to the mesencephalic optic tectum of both Discoglossus and Pleurodeles (Becker et al., 1993). Comparing the PSANCAM immunolabelling reported in the present research with that obtained by Becker et al. (1993) in Pleurodeles by using a rat monoclonal anti PSA-NCAM antibody, similarities either in localizations or in type of labelling were so close as to lead us to envisage the antibody used in our study (a mouse monoclonal anti PSA-NCAM antibody,

Fig. 1 - A) Pleurodeles, stage 30. A patching, strong PSA-NCAM immunolabelling was shown by the wall of the prosencephalon. In this brain coronal section, the deep cephalic flessure, occurring between telencephalon and diencephalon, caused the thalamus and caudal portion of the striatum to be seen in the same section level. D.th.=dorsal thalamus; V.th.=ventral thalamus; $S=$ striatum, scale bar $=100 \mu \mathrm{m}$. B) Ambystoma, stage 36. PSA-NCAM immunopositive cells in the olfactory epithelium (OE). Some faintly stained cells (arrowheads) were also seen in the adjacent olfactory bulb (OB). Black pigment dots were seen throughout the section, scale bar $=10 \mu \mathrm{m}$. C) Pleurodeles, stage 38. A coronal section of the telencephalic hemispheres showing an intense PSA-NCAM-IR mainly localized in the septum (Spt), striatum (S) and olfactory bulb (OB). P=pallium, scale bar=100 $\mu \mathrm{m}$. D) Ambystoma, stage 40. The characteristic PSA-NCAM-IR outlining the periventricular neuron plasma membranes in the optic tectum (OT) and tegmentum of the mesencephalon. Black pigment dots were seen throughout the section, scale bar=10 $\mu \mathrm{m}$. E) Pleurodeles, stage 47. A well distinct PSA-NCAM-IR shown by fiber systems in the posterior pole of the telencephalic hemisphere (DMP=dorsomedial pallium). Positive nerve fibers, likely belonging to the stria medullaris, occurred in the neuropil of the habenular (Hab) nucleus. v=ventricle, scale bar=50 $\mathrm{mm}$. F) Ambystoma, stage 52. A bilateral cluster of PSA-NCAM immunopositive cells in the basal telencephalon (septum). The heavily immunostained fiber forming the terminal nerve (NT) are also well detectable, scale bar $=50 \mu \mathrm{m}$. 

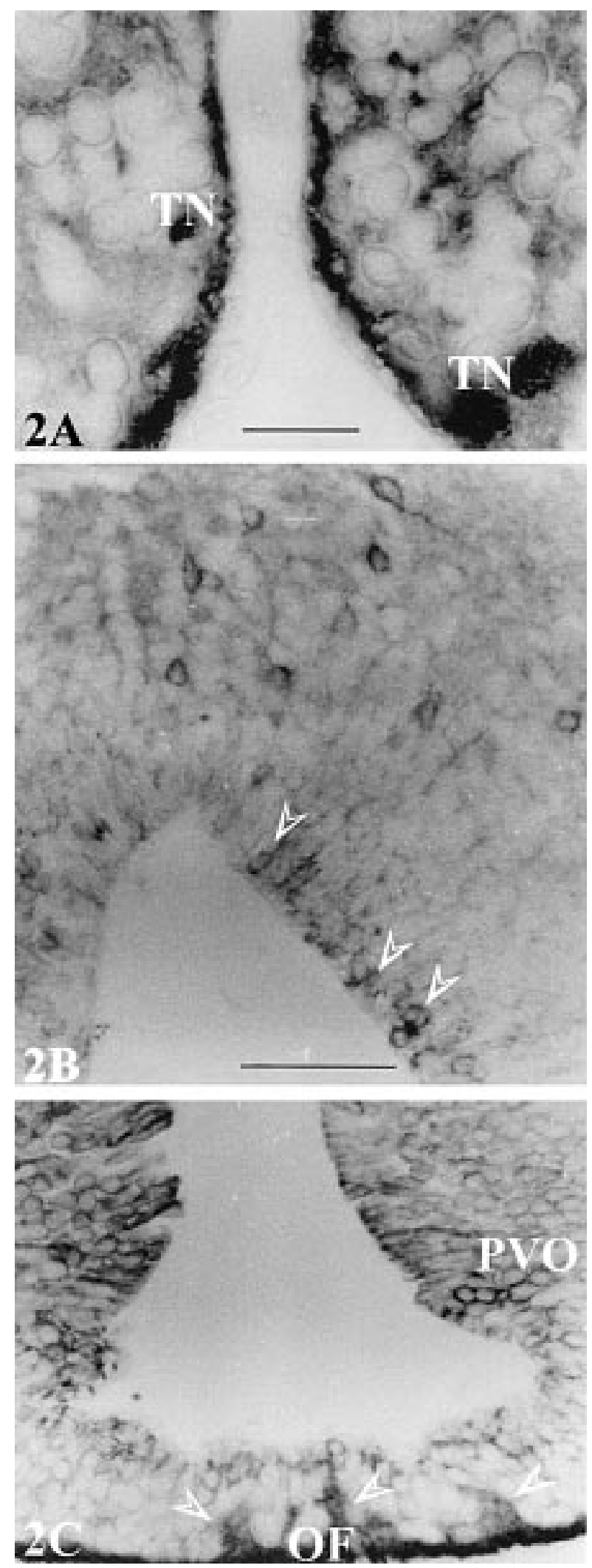

Rougon et al., 1986) as a good marker for urodele developing brain. Moreover, all the controls carried out on brain sections indicate the specificity of the immunolabellings observed.

In the urodele developing brain, the PSANCAM-IR was actually undetectable till stage 28 . By stage 30 the immunolabelling was observed and progressively increased throughout the brain, as reported in other vertebrate groups (see for example, Seki and Arai, 1993). By contrast, close to metamorphosis, the PSA labelling decreased to become restricted to selective areas with remarkable differences between the two species. In Pleurodeles, immunostaining was in fact progressively reduced to the olfactory system in comparison with Ambystoma brain where the PSA-NCAM-IR maintained a more extended distribution (for example through the dorsal pallium and amygdala of the telencephalon) possibly corresponding to a persistence of a widespread neuronal plasticity.

In our study, the distribution of LHRH immunolabelling was almost consistent with that reported in other amphibian species (see, for all, the exhaustive review by Muske, 1993). The earliest m-LHRH-IR detection (stage 30) is contemporary, at least in the olfactory system, with the appearance of PSANCAM immunolabelling. A bit later (stage 38), a well developed neuronal system labelled by both anti LHRH antibodies was seen to extend from the forebrain to the mesencephalic tegmentum in the two species. Numerous LHRH-immunoreactive cells were observed, from early development stages to adult specimens, in the hypothalamus. In particular, by comparing PSA- and LHRH-IRs in serial sections, the two markers were closely localized in the infundibular wall, especially at the middle of metamorphosis (stages 44/45).

Fig. 2 - A) Ambystoma, adult specimen. Tightly assembled PSA-NCAM immunopositive fiber bundles, forming the roots of the terminal nerve (TN), were seen in the septal area of the telencephalon, scale bar=2,5 $\mu \mathrm{m}$. B) Ambystoma, adult specimen. Numerous PSA-NCAM immunopositive polymorphic cells were observed throughout the dorsal pallium of the telencephalon. Positive cells (arrowheads) were also seen in the ependymal and subependymal layers, scale bar $=100 \mu \mathrm{m}$. C) Ambystoma, adult specimen. Suprachiasmatic hypothalamus. PSA-NCAM-IR in the periventricular grey (included the ependymal layer) of the preoptic recess. Some cells of the paraventricular organ (PVO) were immunopositive as well the some bundles of optic fibers (OF, arrowheads), scale bar $=100$ $\mu \mathrm{m}$ 

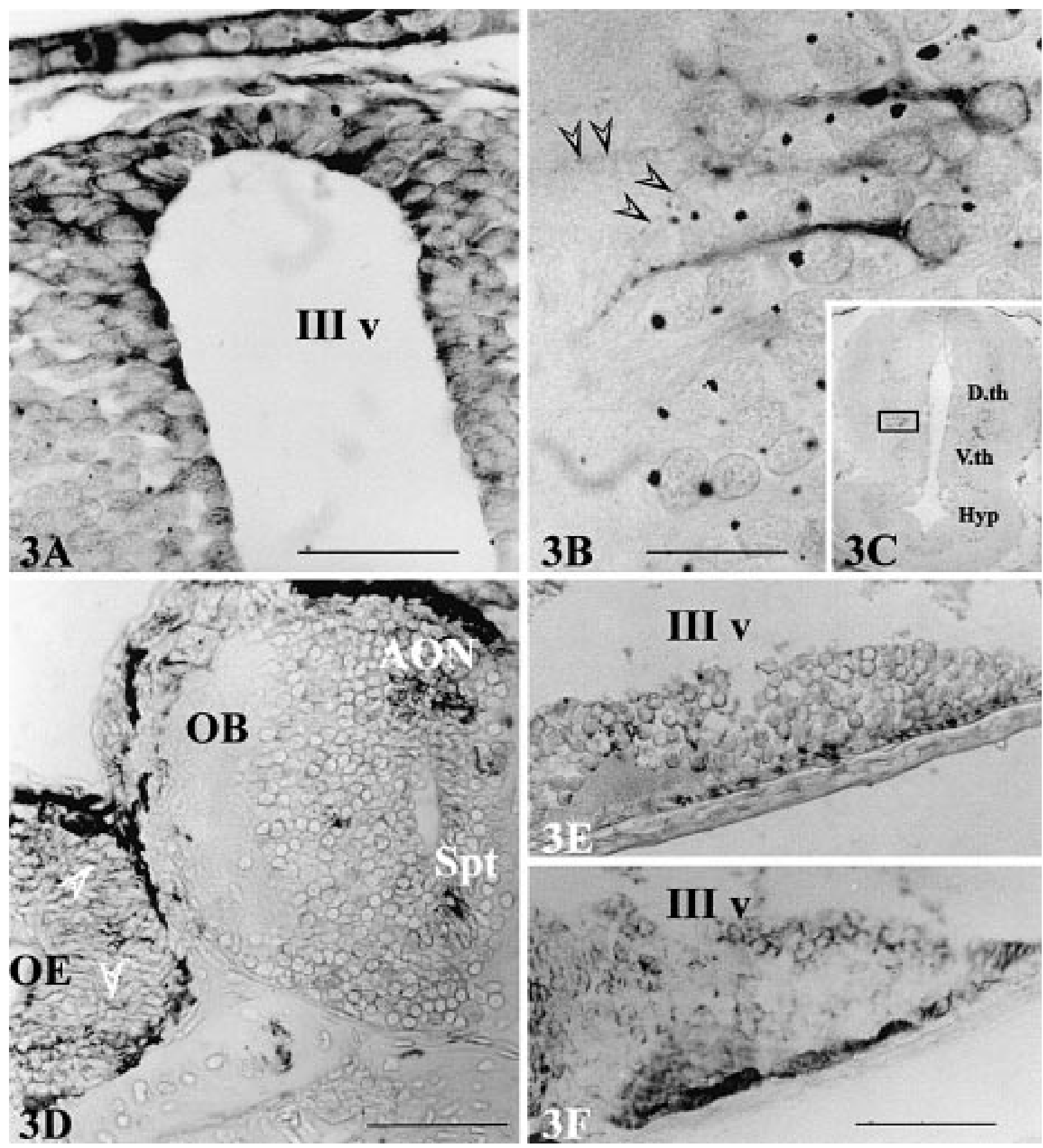

Fig. 3 - A) Ambystoma, stage 38. An abundant m-LHRH-IR in the pretectal area. (III v= third ventricle), scale bar=10 $\mu \mathrm{m}$. B,C) Ambystoma, stage 39. A coronal section of the diencephalon (C, at lower magnification) showing a m-LHRH immunolabelled cluster of tufted neurons placed (in the inset in $\boldsymbol{C}$ ) at the boundary between dorsal (D.th) and ventral thalamus (V.th) and sending their processes (arrowheads) in a ventrolateral direction. Abundant pigment dots were seen throughout the section (Hyp $=$ hypothalamus). Scale bar=10 um. D) Pleurodeles, stage 43. A rostral coronal section of a telencephalic hemisphere showing m-LHRH immunopositive cells in the anterior olfactory nucleus (AON) and septum (Spt). Some labelled cells (arrowheads) were also seen in the olfactory epithelium $(\mathrm{OE})$. Abundant melanophores were outlining both the structures. $\mathrm{OB}=$ olfactory bulb, scale bar=10 $\mu \mathrm{m}$. E,F) Pleurodeles, stage 44. In two sagittal, serial sections of the posterior hypothalamus, the m-LHRH- $(\boldsymbol{E})$ and the PSANCAM-(F) IRs were closely distributed in the ventral wall of the third ventricle (=III v), scale bar $=10 \mu \mathrm{m}$. 

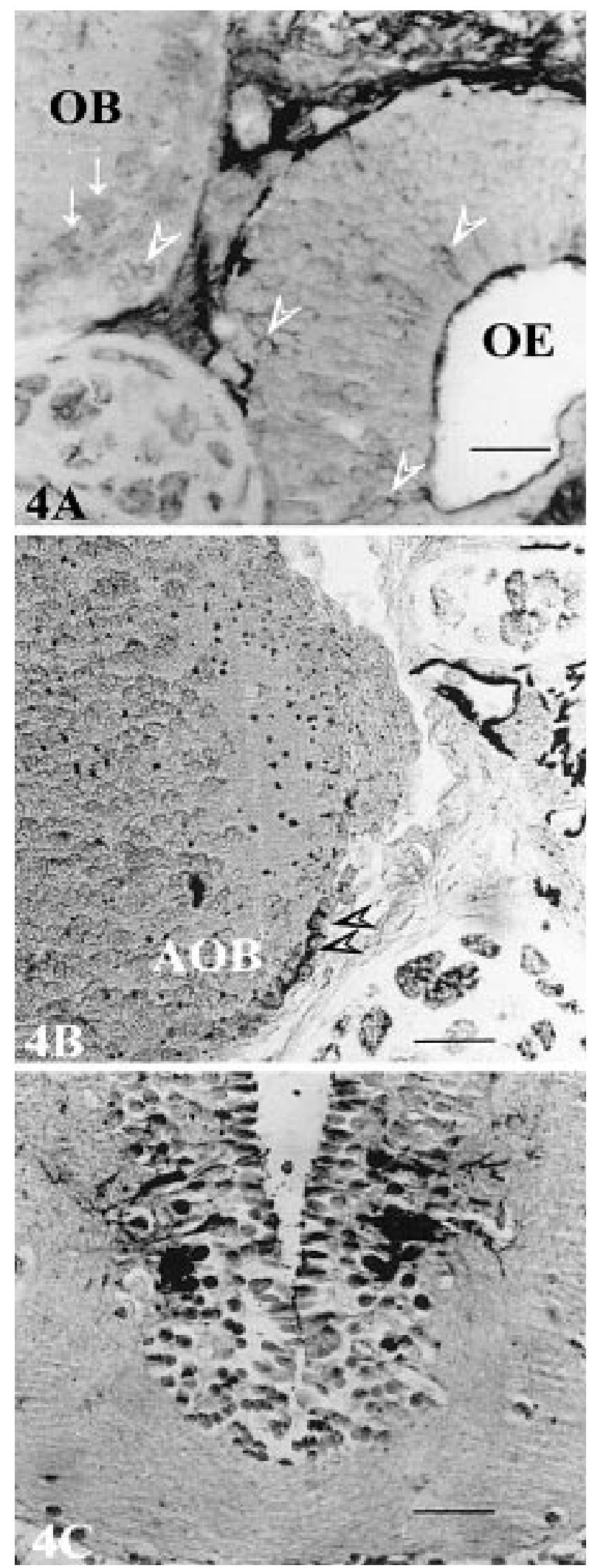

Some peculiarities observed during Ambystoma brain development seem to deserve some comments.

Only in Ambystoma, stage 39, at the boundary between the dorsal and ventral thalamus, have we observed a small group of m-LHRH immunopositive neurons which were not seen later. Since occurrence of transient nervous structures is not unusual during ontogenesis, a transient phenotype may be hypothesized for such m-LHRH containing tufted neurons (although different mechanisms acting in the regulation of neuron number, such as the programmed cell death, can be also envisaged). On the other hand, a direct pathway from the hypothalamus to the dorsal pallium, through the amygdala, was shown in Pleurodeles during the first half of metamorphosis. The posterior part of this tract vanished during the second part of metamorphosis, remaining, in the adult, only its anterior (amygdalo-pallial) portion (Clairambault and Timmel, 1990). Another case of restriction was reported in the olfactory system of the same species (Clairambault and Timmel, 1990). Moreover, a possible transient expression of the caudal LHRH system, lacking in the adult, was also postulated in mammals (Muske, 1993).

In general, our results are basically in agreement with those reported for the developing mouse brain (Schwanzel-Fukuda et al., 1992) and remarkably show close functional relationships between the two markers studied in the present work. It seems likely that, as in rodents, a PSANCAM scaffold formed by fiber systems of, respectively, terminal nerve and optic nerve and tract (which are characterized by an intense labelling long after metamorphosis) plays a crucial role in guiding LHRH containing structures toward their final location in the basal telencephalon and hypothalamic area, respectively.

Fig. 4 - A) Pleurodeles, adult specimen. m-LHRH immunopositive cells (arrowheads) were seen in the olfactory epithelium (OE) as well as, together with nerve fibers (arrows), in the olfactory bulb (OB), scale bar $=50 \mu \mathrm{m}$. B) Pleurodeles, adult specimen. m-LHRH immunopositive cells (arrowheads) in the accessory olfactory bulb (AOB). Pigment dots are abundantly distributed throughout the olfactory bulb, scale bar $=50$ $\mu \mathrm{m}$. C) Ambystoma, adult specimen. A coronal section of the caudal diencephalon showing a bilateral cluster of intensely cII-LHRH immunopositive neurons in the posterior tuberculum, scale bar $=100 \mu \mathrm{m}$. 
Of special interest seem to be the immunostained neurons found in the paraventricular organ or PVO, a circumventricular organ of non-mammals lining the third ventricle. This small nucleus plays a crucial role, in both Anura and Urodela, in courtship behavior in so far as it is directly implicated in the control of neuroendocrine events (Dubé et al, 1990) by way of hormonal synthesis (for example LHRH, Muske et al., 1994 in Taricha granulosa) or monoamine (dopamine and serotonin) release (Beltramo et al., 1998 in Ambystoma). Taken together, the results outline an interesting point. In mammals, a noticeable NCAM PSA-IR has been shown in some hypothalamic regions (Theodosis et al., 1991; Bonfanti et al., 1992) as well as a parallel between the appearance of PSA and dopamine (Schultz and Kimher, 1992). In Ambystoma, the PVO was characterized by PSANCAM-IR, LHRH immunopositive cells (present work) as well as a population of dopaminergic neurons (Beltramo et al., 1998). Therefore multiple neurochemical events converging in the PVO suggest a pivotal role for this nucleus in urodele hypothalamic function, and leads us to envisage for PSA-NCAM a facilitating action in the development of the endocrine reproductive system.

In conclusion, the differences found in PSANCAM-IR during development of the two urodele species will be commented on in the light of some evolutionary considerations. As reported above, the progressive decreasing of PSA-NCAM-IR in Pleurodeles at the end of metamorphosis might correlate with an increasing morphological stability of the nervous tissue (Fields and Itoh, 1996), in contrast to the more extended plasticity conserved by the juvenile and adult Ambystoma brain. Paedomorphosis is considered as a state in which an adult of a taxon exhibits morphological characteristics displayed by juvenile forms of related groups (outgroups) as the result of heterochronic developmental processes (Alberch et al., 1979). The Urodele perennibranchiate species (Proteidea, Sirenidea, Necturidea and Ambystomidea) are paedomorphic, and among Ambystomatidea only Ambystoma is paedomorphic. This species is commonly considered as exhibiting a "light" paedomorphosis with a fully developed nervous system (Roth et al., 1993). Nevertheless, in the light of the present results, by comparing Ambystoma mexicanum and Pleurodeles waltl, we cannot agree with this statement. Our observations have, in fact, shown that at stages 55/56 (and later on) PSA-
NCAM immunopositive cells and fibers were retained only in the olfactory system of Pleurodeles, while in Ambystoma numerous cells were immunoreactive throughout the telencephalon, preoptic area and PVO. Hence, at the end of metamorphosis, only a few adult traits (e.g. the anatomical organization) of the Ambystoma brain, when compared to that of Pleurodeles, are fully achieved. Other characteristics, such as the neuronal patterns, still show a high degree of phenotypic plasticity. Therefore, at least for the brain, Ambystoma mexicanum can be considered as a complete paedomorphic species.

\section{ACKNOWLEDGEMENTS}

The authors gratefully acknowledge Dr. G. Rougon, Dr. R. Benoit and Dr. F. Vandesande for their kind gifts of the antisera, and Mr. Claudio Gendusa and Mr. Franco Scaranari for their technical help in iconography.

Financial support by NATO Collaborative Research Grant n. 970281 (t MFF)

\section{REFERENCES}

Alberch P., Gould S.S., Osten G.F., and Wake D.B.: Size and shape in ontogeny and phylogeny.Paleobiol. 5, 296-317, 1979.

Becker C.G., Becker T., and Roth G.: Distribution of NCAM180 and polysialic acid in the developing tectum mesencephali of the frog Discoglossus pictus and the salamander Pleurodeles waltl. Cell.Tissu. Res. 272, 289-301, 1993.

Beltramo M., Pairault C., Krieger M., Thibault J., Tillet Y., and Clairambault P.: Immunolocalization of aromatic Laminoacid decarboxylase, tyrosine hydroxylase, dopamine and serotonin in the forebrain of Ambystoma mexicanum. J.Comp.Neurol. 391, 227-247, 1998.

Bonfanti L., Olive S., Paulain D.A., and Theodosis D.T.: Mapping on the distribution of polysialylated neural cell adhesion molecule throughout the central nervous system of the adult rat: an immunohistochemical study. Neuroscience 49, 419-436, 1992.

Bonfanti L., and Theodosis D.T.: Expression of polysialylated neural cell adhesion molecule (PSA-NCAM) by proliferating cells in the subependymal layer of the adult rat, in its rostral extension and in the olfactory bulb. Neuroscience 62 , 291-305, 1994.

Clairambault P., and Timmel J.F.: Developmental organization of the amphibian pallium. Exp. Br. Res. 19, 29-41, 1990. 
Collin F., Chartrel N., Fasolo A., Conlon J.M., Vandesande F., Vaudry H.: Distribution of two molecular forms of gonadotropin-releasing hormone $(\mathrm{GnRH})$ in the central nervous system of the frog Rana ridibunda. Brain Res. 703, 111128, 1995.

D’Aniello B., Pinelli C., Di Fiore M.M., Iela L., King J.A., and Rastogi R.K.: Development and distribution of gonadotropin-releasing hormone neuronal systems in the frog (Rana esculenta): immunohistochemical analysis. Dev. Brain Res. 89, 281-288, 1995.

Dubé L., Clairambault P., and Malacarne G.: Striatal afferents in the newt Triturus cristatus. Br. Behav. Evol. 35, 212-226, 1990.

Fields R. D. and Itoh K.: Neural cell adhesion molecules in activity-dependent development and synaptic plasticity. Trends Neurosci. 19, 473-480, 1996.

Gallien L., and Durocher M.: Table chronologique du dévelopment chez Pleurodeles waltlii. Bull. Biol. France Belgique 91(2), 97-114, 1957.

Hayes W. P., Wray S., and Battey J.F.: The frog gonadotropinreleasing hormone-I (GnRH-I) gene has a mammalian-like expression pattern and conserved domains in GnRH-associated peptide, but brain onset is delayed until metamorphosis. Endocrinology 134(4), 1835-1845, 1994.

Hoffman S., and Edelman G.M.: Kinetics of homophilic binding by embryonic and adult forms of the neural cell adhesion molecule. Proc. Natl. Acad. Sci., USA 80, 5762-5766, 1983.

Hoffman S., Chuong C.M., and Edelman G.M.: Evolutionary conservation of the key structures and binding functions of neural cell adhesion molecules. Proc. Natl. Acad. Sci., USA, 81, 6881-6885, 1984.

King J.A., Steneveld A.A., and Millar R.P.: Differential regional distribution of gonadotropin-releasing hormones in amphibian (clawed toad, Xenopus laevis) brain. Regulatory Peptides 50, 277-289, 1994.

Murakami S., Kikuyama S., and Arai Y.: The origin of the luteinizing hormone-releasing hormone (LHRH) neurons in newt (Cynops pyrrhogaster): the effect of olfactory placode ablation. Cell. Tissue Res. 269, 21-27, 1992.

Muske L.E.: Evolution of gonadotropin-releasing hormone (GnRH) neuronal systems. Brain Behav. Evol. 42, 215-230, 1993.

Muske L.E., and Moore F.L.: Antibodies against different forms of GnRH distinguish different populations of cells and axonal pathways in an urodele amphibian, Taricha granulosa. J. Comp. Neurol. 345, 139-147, 1994

Northcutt G.R., and Muske L.E.: Multiple embryonic origins of gonadotropin-releasing hormone (GnRH) immunoreactive neurons. Dev. Brain Res. 78, 279-290, 1994.

Roth G., Nishikawa K.C., Naujoks-Manteuffel C., Schmidt A., and Wake D.B.: Paedomorphosis and simplification in the nervous system of salamanders. Brain Behav. Evol. 42, 137-170, 1993.
Schwanzel-Fukuda M., Abraham S., Crossin K.L., Edelman G.M., Pfaff D.W.: Immunocytochemical demonstration of neural cell adhesion molecule (NCAM) along the migration route of luteinizing hormone-releasing hormone $(\mathrm{LHRH})$ neurons in mice. J. Comp. Neurol. 321, 1-18, 1992

Seki T., and Arai Y.: Distribution and possible roles of the highly polysialylated neural cell adhesion molecule (NCAM$\mathrm{H})$ in the developing and adult central nervous system. Neurosci. Res. 17(4), 265-290, 1993.

Shults C.W., and Kimber T.A.: Mesencephalic dopaminergic cells exhibit increased density of neural cell adhesion molecule and polysialic acid during development. Dev. Br. Res. 65, 161-172, 1992.

Soares S., von Boxberg Y., Ravaille-Veron M., Vincent J.D., and Nothias F.: Morphofunctional plasticity in the adult hypothalamus induces regulation of polysialic acid-neural cell adhesion molecule through changing activity and expression levels of polysialyltransferase. J. Neurosci. 20(7), 2551-7, 2000.

Sunshine J., Balak K., Rutishauser U., and Jacobson M.: Changes in neural cell adhesion molecule (NCAM) structure during vertebrate neural development. Proc. Natl. Acad. Sci. USA. 84, 5986-5990, 1987.

Theodosis D.T., Rougon G., and Paulain D.A.: Retention of embryonic features by an adult neuronal system capable of plasticity: polysialylated neural cell adhesion molecule in the hypothalamo-neurohypophysial system. Proc. Natl. Acad. Sci., USA 88, 5494-5498, 1991.

Van Gils J., Absil P., Grauwels L., Moons L., Vandesande F., Balthazar J. Distribution of luteinizing hormone-releasing hormones I and II (LHRH-I and -II) in the quail and chicken brain as demonstrated with antibodies directed against synthetic peptides. J. Comp. Neurol. 334(2), 304-323, 1993. 